\title{
Diagnosis and Surgical Outcomes of Patients with Anomalous Left Coronary Artery from the Pulmonary Artery: A Single Taiwanese Medical Center Experience
}

\author{
Wan-Fu Hsu, MD, ${ }^{1,2,3}$ Pi-Chang Lee, MD, ${ }^{3,4}$ Hsing-Yuan Li, MD, PhD, ${ }^{3,4}$ Shao-Wei Huang, MD, ${ }^{1,2,3}$ \\ Shyi-Jou Chen, MD, PhD, ${ }^{1}$ Fei-Yi Wu, MD $, 4,6$ Zen-Chung Weng, MD, ${ }^{7}$ Be-Tau Hwang, MD ${ }^{4,5}$ \\ ${ }^{1}$ Department of Pediatrics, Tri-Service General Hospital, National Defense Medical Center, Taipei City, Taiwan, ROC; ${ }^{2}$ Department of \\ Pediatrics, Penghu Branch of Tri-Service General Hospital, National Defense Medical Center, Penghu County, Taiwan, ROC; ${ }^{3}$ Division \\ of Pediatric Cardiology, Children's Medical Center, Taipei Veterans General Hospital, Taipei City, Taiwan, ROC; ${ }^{4}$ School of Medicine, \\ National Yang-Ming University, Taipei City, Taiwan, ROC; 5 Department of Pediatrics, Tungs' Taichung MetroHarbor Hospital, \\ Taichung City, Taiwan, ROC; ${ }^{6}$ Division of Cardiovascular Surgery, Department of Surgery, Taipei Veterans General Hospital, Taipei \\ City, Taiwan, ROC; ${ }^{7}$ Division of Cardiovascular Surgery, Department of Surgery, School of Medicine, College of Medicine, Taipei \\ Medical University Hospital, Taipei City, Taiwan, ROC
}

\section{ABSTRACT}

Background: Anomalous left coronary artery from the pulmonary artery (ALCAPA), a very rare congenital cardiac anomaly, is associated with a high mortality rate among infants who are not diagnosed or treated in a timely manner. Surgical intervention with the reconstruction for a twocoronary-system circulation is the main treatment; however, there have been very few reported cases from Taiwan. In this study, we aim to describe the clinical manifestations, diagnostic methods, surgery types, and surgical outcomes in patients with ALCAPA from a single Taiwanese medical center.

Methods: We retrospectively reviewed patients diagnosed with ALCAPA who underwent surgery at our institution between January 2001 and October 2018. Clinical presentations, noninvasive and invasive study results, surgical methods, and postoperative follow-up results were assessed from medical records. Moreover, literature on this particular cardiovascular anomaly was reviewed.

Results: The study included 6 patients $(5$ children and 1 adult). The diagnosis was confirmed using cardiac catheterization and coronary angiography in four patients and only echocardiography in two patients. All patients underwent surgical correction and survived. Four patients showed improvements in left ventricular function and mitral regurgitation (MR).

Conclusion: Early diagnosis and timely surgical intervention could avoid mortality regardless of the method of operation. ALCAPA can be definitively diagnosed using noninvasive echocardiography. Both left ventricular systolic function and mitral insufficiency could improve after the surgical intervention in pediatric patients. Repair or replacement of the mitral valve could be reserved for persistent MR complicated with

Received August 7, 2019; accepted December 4, 2019.

Correspondence: Be-Tau Hwang, MD, FACC, Tungs' Taichung MetroHarbor Hospital, No.699, Sec. 8, Taiwan Blvd., Taichung City 435, Taiwan, ROC; +886-4-26581919; fax: +886-4-26582193; (e-mail: btbwang@vghtpe.gov.tw). congestive heart failure, particularly in patients who received the initial operation beyond infancy.

\section{INTRODUCTION}

Anomalous left coronary artery from the pulmonary artery (ALCAPA), a very rare congenital cardiovascular anomaly, was first reported in an anatomical study by Brooks in 1885 [Brooks 1885]. Bland et al described the clinical features of this anomaly in 1933, and then this anomaly was referred to by the eponym Bland-White-Garland syndrome [Bland 1933]. Its incidence has been estimated at 1 in 300,000 live births; it accounts for $0.24 \%-0.46 \%$ of all congenital cardiovascular anomalies [Yau 2011; Formica 2014].
A

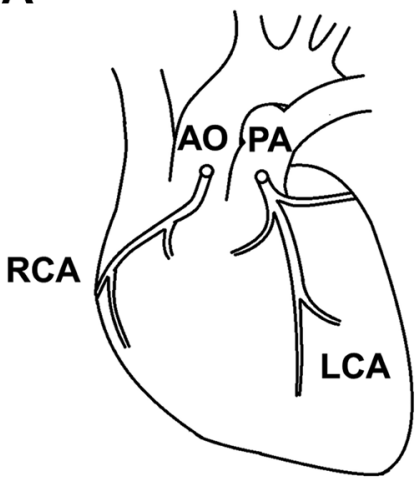

B

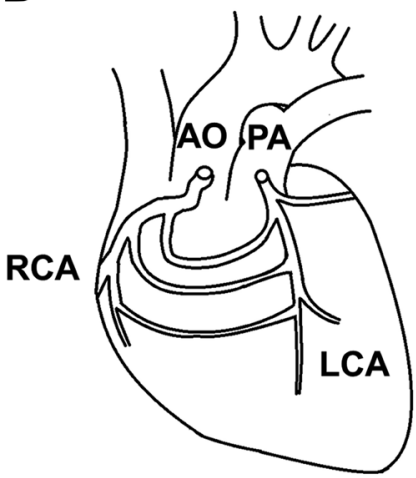

Figure 1. Diagram of ALCAPA. (A) If there is no sufficient collateral developed between the RCA and LCA, the myocardium of the LV will be perfused with low oxygen-containing blood at low pressure and myocardial ischemia with severe congestive heart failure can develop over time. (B) If there are sufficient collaterals between the RCA and LCA, patients with ALCAPA may survive beyond their childhood. AO, aorta; LCA, left coronary artery; PA, pulmonary artery; RCA, right coronary artery; LV, left ventricle. 

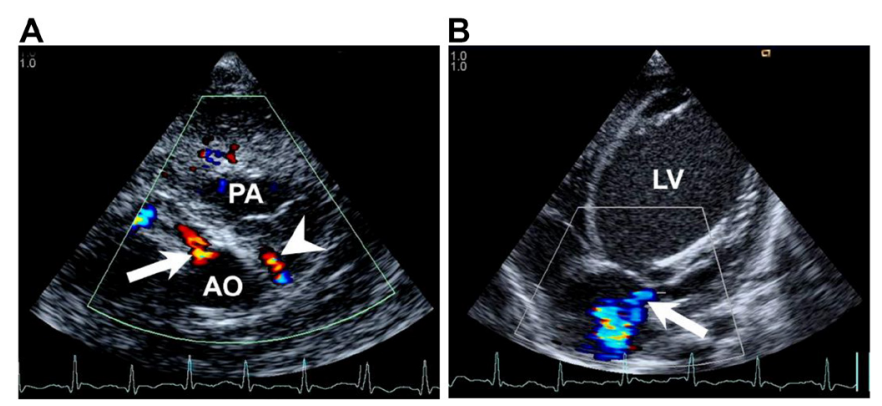

Figure 2. Transthoracic echocardiography of ALCAPA. (A) Parasternal short-axis view showing the LCA connected to PA with retrograde flow above the pulmonary valve (arrowhead) and dilated RCA (arrow). (B) Apical four-chamber view showing dilated LV, significant mitral regurgitation (arrow), subendocardial fibroelastosis, and enhanced papillary muscle echogenicity. AO, aorta; LV, left ventricle; PA, pulmonary artery; LCA, left coronary artery; RCA, right coronary artery.

At the intrauterine stage, fetuses with ALCAPA typically are asymptomatic; however, after birth, along with a fall in PA pressure and pulmonary vascular resistance, the perfusion pressure in the anomalous left coronary artery (LCA) decreases [Formica 2014; Jiang 2014]. Thus, the myocardium of the left ventricle (LV) is perfused with low oxygen-containing blood at low pressure (Figure 1A), which predisposes patients to ischemia and even infarction over the anterolateral myocardium of the LV [Lardhi 2010]. Both mitral regurgitation (MR) and congestive heart failure $(\mathrm{CHF})$ can develop over time. In infants with ALCAPA, the typical symptoms and signs of CHF include feeding difficulties, irritability, diaphoresis, tachypnea, and tachycardia. Chest pain might be misdiagnosed as infantile colic [Pachon 2014]. In patients without an early diagnosis and surgical intervention, the mortality rate associated with severe $\mathrm{CHF}$ during the first year of life may be as high as 90\% [Yau 2011; Pachon 2014]. Interestingly, if sufficient numbers of collateral vessels develop between the right and left coronary arteries (Figure 1B), patients might survive beyond childhood without significant symptoms, except a systolic or continuous murmur [Yau 2011]. However, some adult patients might present with dyspnea, chest pain, syncope, exercise intolerance, and even sudden cardiac death because of acute myocardial ischemia or infarction during exercise or malignant ventricular arrhythmia [Yau 2011; Pachon 2014].

Traditionally, the diagnosis of ALCAPA was established by cardiac catheterization and coronary angiography [Yau 2011]. However, noninvasive diagnostic modalities, including echocardiography and computed tomography angiography, recently have been reported as alternative and accurate approaches [Yau 2011; Pachon 2014]. The definite treatment of ALCAPA is surgical intervention for adequate coronary circulation, and several methods have been developed for such an intervention, which can be classified as either oneor two-coronary-system repair [Peña 2009; Zheng 2010]. One-coronary-system repair involves the ligation of the abnormal LCA origin; however, because of the high incidence of complications, such as ALCAPA recanalization,
A

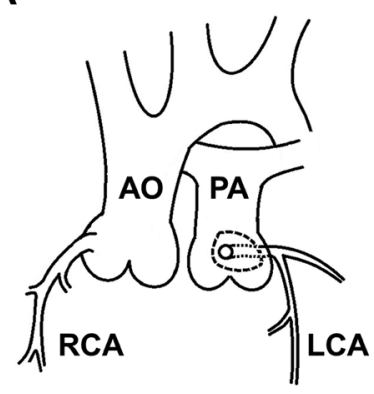

B

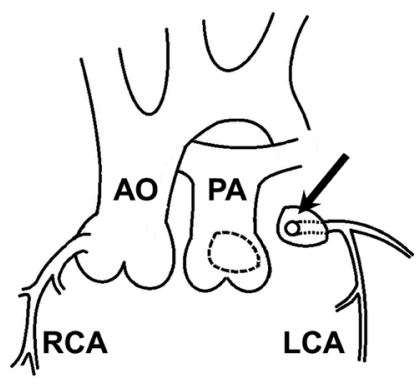

C
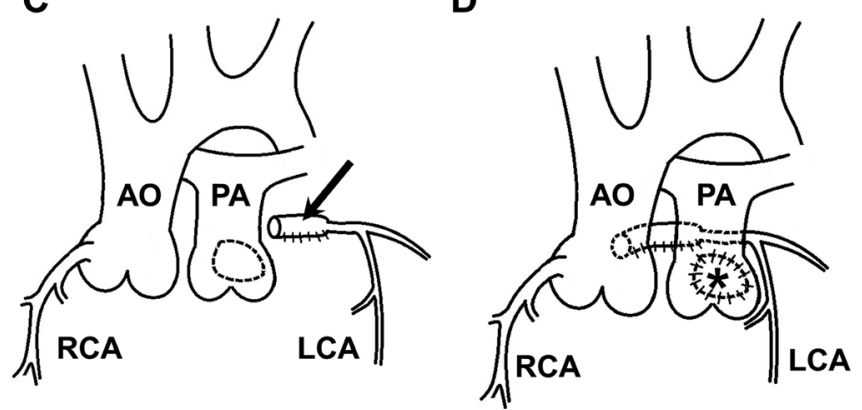

Figure 3. Surgical reconstruction of the two-coronary-system circulation with direct re-implantation of the LCA to the AO. (A) The orifice of the anomalous LCA was excised using a generous cuff of the PA wall. (B \& C) The PA wall flap was used as a tube material to connect the LCA and AO (arrow). (D) The LCA was anastomosed to the AO, and the PA wall was repaired with an autologous pericardial patch (asterisk). $\mathrm{AO}$, aorta; LCA, left coronary artery; PA, pulmonary artery; RCA, right coronary artery.

atherosclerosis, and sudden death, it has been abandoned and two-coronary-system repair is now universally preferred and accepted [Rodriguez-Gonzalez 2015; Muzaffar 2014; Gao 2017]. Reconstruction for dual coronary circulation can be performed by the direct reimplantation of the anomalous LCA into the ascending aorta, creation of an aorticopulmonary tunnel (also known as the Takeuchi procedure), or use of a venous or arterial bypass graft to connect the aorta and proximal left anterior descending artery [Peña 2009].

There are very few reports on ALCAPA in Taiwanese population; there have been only case reports regarding the congenital anomaly in the past 10 years [Huang 2017; Tseng 2013; Hsu 2014; Jin 2011; Su 2010]. This retrospective study provides a more comprehensive review of ALCAPA in a Taiwanese population by assessing the clinical manifestations, diagnostic methods, surgery types, and follow-up results in patients with ALCAPA over the last 17 years from Taipei Veterans General Hospital, a medical center in Taiwan.

\section{MATERIALS AND METHODS}

We retrospectively reviewed the medical records of patients diagnosed with ALCAPA and operated on at Taipei Veterans General Hospital between January 2001 and October 2018. 
Table 1. Patient data (characteristics before the operation, diagnostic methods, surgical methods, and outcomes)

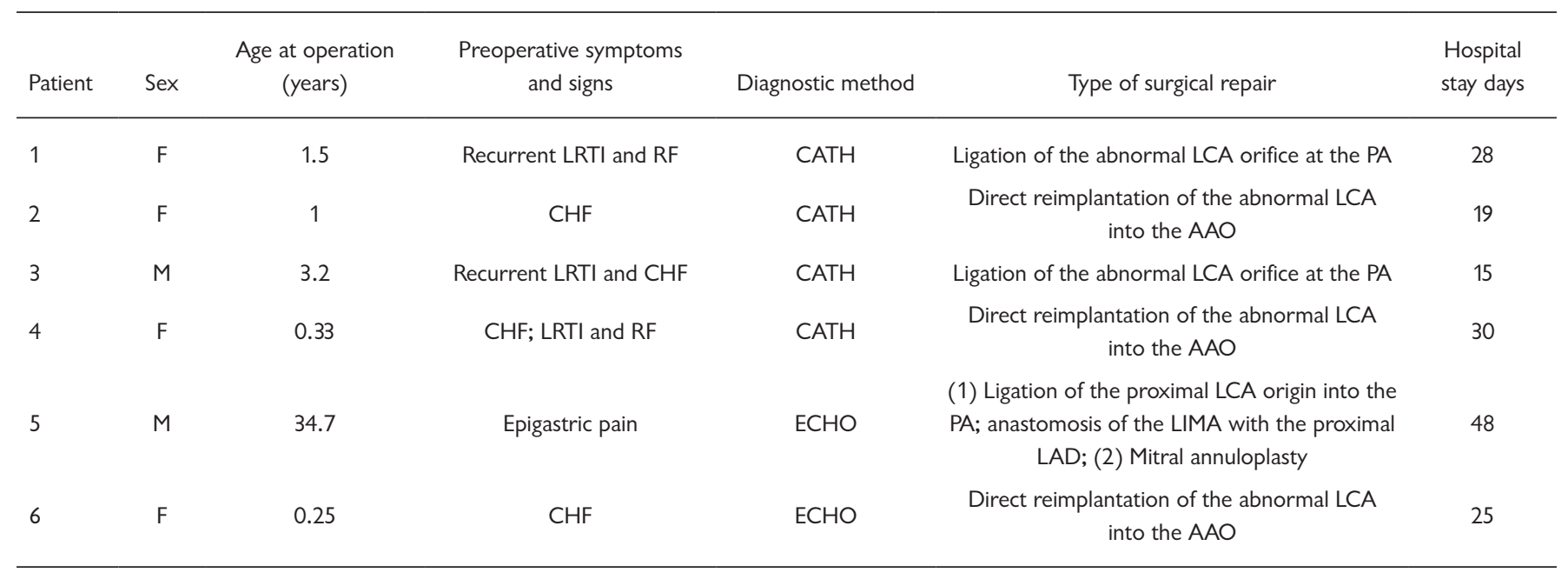

AAO, ascending aorta; CATH, cardiac catheterization; CHF, congestive heart failure; ECHO, echocardiography; F, female; LRTI, lower respiratory tract infection; $R F$, respiratory failure; LCA, left coronary artery; LIMA, left internal mammary artery; LAD, left anterior descending artery; M, male; PA, pulmonary artery

We assessed the patients' sex, age, weight at diagnosis, clinical manifestations, diagnostic methods, surgery types, and late follow-up findings.

During each time of follow-up, after the surgical correction of ALCAPA, we routinely performed history taking for symptoms and signs, physical examinations, and echocardiographic evaluations. Echocardiography was especially focused on the left ventricular ejection fraction (LVEF) and MR grade. Furthermore, postoperative medical records regarding outpatient department visits, emergency department visits, and hospitalization were reviewed to evaluate relevant symptoms and signs, medications, and surgical interventions.

We performed data entry in a Microsoft Excel file; however, the rarity of the cases limited the analysis that reasonably could be performed. The descriptive data are presented as numbers and percentages. This study was approved by the Institutional Ethical Review Board of Taipei Veterans General Hospital (VGHTPE-IRB No. 2019-04-013BC); the requirement for informed consent was waived owing to the retrospective nature of this study.

\section{RESULTS}

The study included 5 pediatric patients (mean age: 1.2 years) and 1 adult patient (age: 34.7 years). All 5 pediatric patients presented with symptoms and signs of CHF, including respiratory dyspnea, recurrent respiratory infections, and failure to thrive in early infancy. One pediatric patient had cardiomegaly since the age of 7 months; however, the patient did not undergo further examination until the age of 3 years when he was referred to our institution. The adult patient presented with nonspecific epigastric pain and was found to incidentally have $L V$ akinesia via echocardiography. Table 1 lists the patients' data, including clinical manifestations, diagnostic methods, surgery types, hospital stay length, and follow-up duration. The surface 12-lead electrocardiograms of all patients revealed different severities of myocardial damage over the anterior or left lateral wall with an abnormal Q wave in $83 \%$ of the patients and significant ST-T wave changes in all patients. All patients had varying degrees of ventricular dysfunction and MR on echocardiography. Figure 2 demonstrates the echocardiographic findings of patient 6 . Both one- and two-coronary-system repair were performed in our patients. Two-coronary-system repair was performed with direct reimplantation of the abnormal LCA to the aorta (Figure 3); however, if the LCA had an inadequate length for reimplantation, one-coronary-system repair was performed with simple obliteration of the LCA opening into the PA. None of the pediatric patients underwent mitral valve repair or replacement at the initial surgical reconstruction.

During the early ( $<30$ days) postoperative period, there were no significant complications after surgical reconstruction; however, one pediatric patient developed acute renal failure and gradually recovered without sequelae after temporal peritoneal dialysis. All pediatric patients showed improvement in left ventricular systolic function on follow-up echocardiography. Table 2 lists the preoperative and late follow-up LVEF and MR in each patient. Patient 2 underwent mitral valve replacement 11 months after the ALCAPA surgery owing to persistent moderate-to-severe MR complicated with CHF. All pediatric patients are currently asymptomatic. The adult patient developed acute pulmonary edema 2 months after the surgery. The recanalization of the opening of the proximal LCA in the PA was noted; patch closure of the opening of the proximal LCA was performed later. Sequential cardiac catheterization revealed total occlusion of the graft 4 years later. Ventricular function did not significantly improve after the surgery, and the patient was hospitalized 4 times owing to CHF and acute pulmonary edema. 
Table 2. Preoperative and late follow-up LVEF and MR in each patient

\begin{tabular}{lccccc}
\hline Patient & Perioperative LVF (\%) & Late follow-up LVEF (\%) & MR severity before the operation & MR severity at late follow-up & Follow-up (months) \\
\hline 1 & 48 & 72 & Minimal & None & 174 \\
2 & $-*$ & 76 & Moderate & Mild & Moderate-to-severe \\
3 & 32 & 65 & Severe & Minimal & Mild (post MVA) \\
4 & 30 & 77 & Mild-to-moderate & Severe \\
5 & 33 & 43 & Moderate & 110 \\
\hline
\end{tabular}

\footnotetext{
*The preoperative data for patient 2 is missing.

**Patient 3 underwent mitral valve replacement 11 months after the ALCAPA surgery.

ALCAPA, anomalous left coronary artery from the pulmonary artery; LVEF, left ventricular ejection fraction; MR, mitral regurgitation; MVA, mitral valve annuloplasty
}

\section{DISCUSSION}

This retrospective descriptive study demonstrated that early diagnosis and suitable surgical intervention were beneficial with regard to overall survival among patients with ALCAPA. To our knowledge, this is the first study to describe the outcome involving 3 methods of surgical intervention in Taiwanese patients with ALCAPA. The postoperative LVEF and MR severity improved in almost all pediatric patients. Echocardiography was useful for both establishing the diagnosis and evaluating cardiac function before and after surgery in patients with ALCAPA.

In pediatric patients with ALCAPA, the most common presentations are poor exercise tolerance, poor weight gain, poor feeding, and tachypnea [Brotherton 2008]. Here, more than half of the pediatric patients had recurrent respiratory tract symptoms or infection before diagnosis. Complete cardiovascular examinations, including cardiac imaging and functional evaluation, may be necessary in infants with recurrent respiratory symptoms or infection. In adult patients, Yau et al reviewed 151 cases and reported that the mean age was 41 years and the most common symptoms were angina, dyspnea, palpitations, and fatigue [Yau 2011]. In our study, the initial presentation of the adult patient was nonspecific, and this might be explained by the relatively young age (34.7 years).

Previously, the diagnosis of ALCAPA was exclusively confirmed by cardiac catheterization and angiography, which shows the entire course of the anomalous coronary artery [Peña 2009]. However, these examinations are invasive and associated with risks and possible complications [Harold 2013]. As technology continues to improve, accurate echocardiographic diagnosis might become possible [Pachon 2014; Ramírez 2011].

The echocardiographic findings of ALCAPA include direct visualization of the connection between the PA and LCA; retrograde flow above the pulmonary valve toward the left and posterior wall of the PA; a dilated right coronary artery; a dilated $\mathrm{LV}$ with systolic dysfunction; significant MR; and abundant color flow Doppler signals within the interventricular septum owing to numerous coronary collaterals, subendocardial fibroelastosis, and enhanced papillary muscle echogenicity [Zheng 2010; Rodriguez-Gonzalez 2015; Yang 2018]. In our study, the first 4 pediatric patients were definitively diagnosed using cardiac catheterization. The subsequent 2 patients ( 1 adult and 1 infant) were diagnosed using only echocardiography.

The definitive treatment for ALCAPA is surgical correction of the abnormal coronary circulation. Currently, establishing a two-coronary-system circulation is the favored surgical approach because it is believed to be associated with reduced morbidity and mortality [Ramírez 2011; Ojala 2010; Vossler 2012]. Nevertheless, the randomized controlled trials to compare outcomes of different surgical methods still are lacking. Both one- and two-coronary-system approaches were performed in our patients, and the overall survival and LVEF improvement during the follow-up period were satisfactory in all the pediatric patients. The postoperative LVEF of the adult patient did not improve, which might be explained by chronic myocardial ischemia. However, owing to the risk of sudden death, timely surgical intervention is required in adult patients with ALCAPA [Rajbanshi 2014]. Furthermore, the recanalization of the anomalous LCA opening into the $\mathrm{PA}$ and obliteration of the graft were noted 2 months later. Therefore, establishing a normal anatomical configuration by the direct reimplantation of the anomalous LCA into the ascending aorta in adult patients might help avoid these complications [Rajbanshi 2014]. However, the optimal method of surgical repair in adult patients with ALCAPA still is inconclusive [Vossler 2012].

Although MR is common in patients with ALCAPA [Ramírez 2011; Ojala 2010; Alexi-Meskishvili 2011], repair or replacement of the mitral valve is a controversial procedure for ALCAPA correction [Talwar 2013]. The mechanisms of MR are usually multifactorial, including myocardial ischemia or infarction, papillary muscle dysfunction because of scarring and calcification, mitral ring dilatation with papillary muscle displacement, dyskinesia of the LV-free wall, and endocardial fibroelastosis involving the chordae tendineae, papillary 
muscles, and mitral leaflets [Ojala 2010; Azakie 2003]. Some studies have suggested that the mitral valve should not be repaired or replaced during the first attempt to reconstruct the coronary circulation because MR usually improves over time after surgery [Gao 2017; Ramírez 2011; Ojala 2010; Alexi-Meskishvili 2011; Azakie 2003]. Furthermore, mitral valve repair or replacement might increase operative difficulty and procedure time [Muzaffar 2014; Ben 2009]. However, regardless of the MR degree, Isomatsu et al. recommended performing simultaneous mitral annuloplasty at the anterolateral commissure, which was effective for reducing regurgitation and was not a very time consuming procedure [Isomatsu 2001]. None of our pediatric patients underwent mitral valve annuloplasty during the initial ALCAPA surgery, and MR improved during follow-up in 3 pediatric patients (60\%). However, 1 pediatric patient required mitral valve replacement 11 months later owing to persistent moderateto-severe MR complicated with CHF. Compared with the other pediatric patients, this patient had more severe MR before the surgical correction. Moreover, this patient underwent the operation at the age of 3.2 years, whereas the other patients were operated on in infancy.

The present study has some strengths. First, the published reports on an ALCAPA in Taiwanese population are rare, and the outcome of different surgical intervention is unclear; the present study described the presentations and surgical outcomes of different methods in Taiwanese patients with ALCAPA. Second, we summarized the associated literature and the echocardiographic findings of ALCAPA. We believe that the results might help in managing patients with ALCAPA.

The primary limitation of this study is that the number of cases was too low for statistical analysis; however, ALCAPA is a very rare congenital cardiac anomaly. To the best of our knowledge, the actual incidence and postoperative prognosis in Taiwanese patients remain undocumented. Further studies involving more cases that statistically can be analyzed are required to support specific diagnostic investigations or surgical approaches. Another limitation of this study is its retrospective design; however, as ALCAPA is extremely rare, a prospective study might require the involvement and cooperation of many institutions.

In conclusion, this study describes both pediatric and adult patients with ALCAPA in Taiwan. Moreover, we discussed 3 methods of surgical intervention and believe that it provides a more comprehensive view of the congenital anomaly. With the low birth rate and the introduction of advanced fetal echocardiographic evaluation in Taiwan, the number of individuals with congenital cardiac anomalies is decreasing. Early diagnosis and timely surgical intervention are crucial to prevent infant mortality associated with correctable congenital cardiac anomalies. ALCAPA can be definitively diagnosed using detailed echocardiographic examination rather than conventional cardiac catheterization. After surgical intervention, clinical symptoms, myocardial functioning, and postoperative survival might dramatically improve in pediatric patients. Although MR commonly is associated with ALCAPA before and after surgery, repair or replacement of the mitral valve might be best reserved for patients with persistent regurgitation complicated with CHF, especially when the initial correction is performed after infancy.

\section{REFERENCES}

Alexi-Meskishvili V, Nasseri BA, Nordmeyer S, et al. 2011. Repair of anomalous origin of the left coronary artery from the pulmonary artery in infants and children. J Thorac Cardiovasc Surg 142:868-74.

Azakie A, Russell JL, McCrindle BW, et al. 2003. Anatomic repair of anomalous left coronary artery from the pulmonary artery by aortic reimplantation: Early survival, patterns of ventricular recovery and late outcome. Ann Thorac Surg 75:1535-41.

Ben Ali W, Metton O, Roubertie F, et al. 2009. Anomalous origin of the left coronary artery from the pulmonary artery: late results with special attention to the mitral valve. Eur J Cardiothorac Surg 36:244-9.

Bland EF, White PD, Garland J. 1933. Congenital anomalies of the coronary arteries: Report of an unusual case associated with cardiac hypertrophy. Am Heart J 8:787-801.

Brooks HSJ. 1885. Two cases of an abnormal coronary artery of the heart, arising from the pulmonary artery: with some remarks upon the effect of this anomaly in producing cirsoid dilatation of the vessels. Trans Acad Med Irel 3:447-9.

Brotherton H, Philip RK. 2008. Anomalous left coronary artery from pulmonary artery (ALCAPA) in infants: A 5 -year review in a defined birth cohort. Eur J Pediatr 167:43-6.

Formica F, Amigoni P, Mariani S, et al. 2014. A rare case of ALCAPA and rheumatic mitral valve regurgitation in an adult patient. Heart Surg Forum.17:E250-52.

Gao Y, Zhang J, Huang GY, et al. 2017. Surgical outcomes of anomalous origin of the left coronary artery from the pulmonary artery in children : An echocardiography follow-up. Chin Med J (Engl) 130:2333-8.

Harold JG, Bass TA, Bashore TM, et al. 2013. ACCF/AHA/SCAI 2013 update of the clinical competence statement on coronary artery interventional procedures: a report of the American College of Cardiology Foundation/American Heart Association/American College of Physicians Task Force on Clinical Compete. J Am Coll Cardiol 62:357-96.

Hsu HT and Wu MT. 2014. Bland-White-Garland syndrome. QJM 108:71-2.

Huang CH, Lin YC, Tsai CS, et al. 2017. Aborted sudden cardiac death in a young male with anomalous left coronary artery arising from the pulmonary artery. J Med Sci 37:256-8.

Isomatsu Y, Imai Y, Shin'oka T, et al. 2001. Surgical intervention for anomalous origin of the left coronary artery from the pulmonary artery: the Tokyo experience. J Thorac Cardiovasc Surg121:792-7.

Jiang GP, Wang HF, Gong FQ, et al. 2014. Diagnostic value of parasternal pulmonary artery short-axis view for the anomalous origin of the left coronary artery from the pulmonary artery. J Cardiol 63:444-8.

Jin YD, Hsiung MC, Tsai SK, et al. 2011. Successful intraoperative identification of an anomalous origin of the left coronary artery from the pulmonary artery using real time three-dimensional transesophageal echocardiography. Echocardiography 28:149-51.

Lardhi AA. 2010. Anomalous origin of left coronary artery from pulmonary artery: A rare cause of myocardial infarction in children. J Fam Community Med 17:113-6. 
Muzaffar T, Ganie FA, Swamy SG, et al. 2014. The surgical outcome of anomalous origin of the left coronary artery from the pulmonary artery. Int Cardiovasc Res J 8:57-60.

Ojala T, Salminen J, Happonen JM, et al. 2010. Excellent functional result in children after correction of anomalous origin of left coronary artery from the pulmonary artery--a population-based complete followup study. Interact Cardiovasc Thorac Surg 10:70-5.

Pachon R, Bravo C, Niemiera M. 2014. Anomalous origin of the left coronary artery from pulmonary artery (ALCAPA). Clin Exp Cardiol 5:10-2.

Peña E, Nguyen ET, Merchant N, et al. 2009. ALCAPA syndrome: Not just a pediatric disease. RadioGraphics 29:553-65.

Rajbanshi BG, Burkhart HM, Schaff HV, et al. 2014. Surgical strategies for anomalous origin of coronary artery from pulmonary artery in adults. J Thorac Cardiovasc Surg148:220-4.

Ramírez S, Curi-Curi PJ, Calderón-Colmenero J, et al. 2011. Outcomes of coronary reimplantation for correction of anomalous origin of left coronary artery from pulmonary artery. Rev Esp Cardiol 64:681-7.

Rodriguez-Gonzalez M, Tirado AM, Hosseinpour R, et al. 2015. Anomalous origin of the left coronary artery from the pulmonary artery: Diagnoses and surgical results in 12 pediatric patients. Texas Hear Inst J 42:350-6.
Su CS, Tsai IC, Lin WW, et al. 2010. Usefulness of multidetector-row computed tomography in diagnosis of anomalous origin of left coronary artery arising from the pulmonary artery. J Chinese Med Assoc 73:492-5.

Talwar S, Jha AJ, Choudhary SK, et al. 2013. Repair of anomalous left coronary artery from pulmonary artery (ALCAPA) beyond infancy. Heart Surg Forum 16:214-20.

Tseng KFY. 2013. Anomalous origin of left coronary artery from the pulmonary trunk in a mildly symptomatic adult female. Case Rep Surg 2013:1-4.

Vossler JD, Entrikin DW, Quartermain MD, et al. 2012. Anomalous left coronary artery from the pulmonary arteryin an adult. Heart Surg Forum $15: 284$.

Yang H, Li J, Ji X. 2018. Diagnosis of anomalous origin of the left coronary artery from the pulmonary artery with echocardiography and digital subtraction angiography. Case Reports Cardiol 2018:1-6.

Yau JM, Singh R, Halpern EJ, et al. 2011. Anomalous origin of the left coronary artery from the pulmonary artery in adults: A comprehensive review of 151 adult cases and a new diagnosis in a 53-year-old woman. Clin Cardiol 34:204-10.

Zheng JY, Han L, Ding WH, et al. 2010. Clinical features and long-term prognosis of patients with anomalous origin of the left coronary artery from the pulmonary artery. Chin Med J (Engl) 123:2888-94. 